\title{
The Fourier-Kelvin Stellar Interferometer (FKSI) nulling testbed II: closed-loop path length metrology and control subsystem
}

\author{
B.J. Frey*, R.K. Barry, W.C. Danchi, T.T. Hyde, K.Y. Lee, A.J. Martino, M.S. Zuray \\ NASA Goddard Space Flight Center, Greenbelt, MD 20771
}

\begin{abstract}
.
The Fourier-Kelvin Stellar Interferometer (FKSI) is a mission concept for an imaging and nulling interferometer in the near to mid-infrared spectral region (3-8 microns), and will be a scientific and technological pathfinder for upcoming missions including TPF-I/DARWIN, SPECS, and SPIRIT. At NASA's Goddard Space Flight Center, we have constructed a symmetric Mach-Zehnder nulling testbed to demonstrate techniques and algorithms that can be used to establish and maintain the $10^{4}$ null depth that will be required for such a mission. Among the challenges inherent in such a system is the ability to acquire and track the null fringe to the desired depth for timescales on the order of hours in a laboratory environment. In addition, it is desirable to achieve this stability without using conventional dithering techniques. We describe recent testbed metrology and control system developments necessary to achieve these goals and present our preliminary results.
\end{abstract}

Keywords: nulling interferometer, symmetric Mach-Zehnder, metrology, closed-loop, extrasolar planets

\section{INTRODUCTION}

The Fourier-Kelvin Stellar Interferometer (FKSI) is an imaging and nulling interferometer mission that will study a broad range of astronomical phenomena. Its science goals include: (1) directly detect extrasolar giant planets (EGP), (2) study debris disks and the evolution of protostellar and evolved stellar systems, and (3) facilitate the study of extragalactic star formation regions and the extended neighborhoods of active galactic nuclei. The angular resolution of FKSI (41 mas) and spectral resolving power $(\lambda / \Delta \lambda \sim 25)$ will allow study of the environmental conditions of EGPs, their atmospheric compositions, and evolutionary history of the planetary systems. In addition, the study of stellar environments will allow observations of the formative stages of planetary systems, protoplanetary disk structure, the evolution of primordial exozodiacal dust, and debris disks about various stellar types. These observations may then be used to determine the characteristics of circumstellar material that could lead to planet formation or the evolution of the habitable zone about post-main sequence stars.

The current mission design concept is a result several studies exploring a variety of beam combination techniques and array architectures. These design studies were conducted initially at GSFC's Instrument Synthesis and Analysis Laboratory (ISAL) and the Integrated Mission Design Center (IMDC) - important functions within GSFC's infrastructure used to facilitate rapid vetting of various mission and instrument design concepts. These studies were then augmented by the work of a larger, focused team of experienced scientists and engineers dedicated to the FKSI mission. The resulting design is a nulling interferometer configuration with an optical system consisting of two $0.5 \mathrm{~m}$ telescopes on a $12.5 \mathrm{~m}$ boom feeding a symmetric Mach-Zehnder beam combiner. The beam combiner includes a null tracker and key technologies currently under development such as high precision cryogenic delay lines and cryogenic optical fibers for wavefront cleanup. More detailed discussions of the mission trade studies and science drivers have been published ${ }^{1,2}$, and mission concept status and updates can be found in a companion paper in this conference ${ }^{3}$.

\section{FKSI TESTBED STATUS}

In order to demonstrate the techniques of nulling and fringe tracking and to investigate additional technologies such as ditherless quadrature phase detection (DQPD) and hollow-glass waveguide (HGW) wavefront cleanup, we have constructed a symmetric Mach-Zehnder interferometer testbed similar in design to that anticipated in the FKSI beam combiner. The testbed (see figures 1 and 2) has been constructed in the Horizontal Flow Facility at NASA's Goddard

\footnotetext{
*Brad.Frey@nasa.gov, phone 1-301-286-7787, FAX 1-301-286-0204
} 
Space Flight Center in Greenbelt, MD; the details of the testbed have been described previously.' The testbed was initially configured with COTS Al reflective optics and a visible $(633 \mathrm{~nm}$ HeNe) laser and later reconfigured with unprotected gold reflective optics and $50 / 50 \mathrm{IR}$ beamsplitters. We have also recently migrated to a $1.15 / 3.39 \mu \mathrm{m} \mathrm{HeNe}$ laser and anticipate the addition of a $1200 \mathrm{~K}$ blackbody in the near term that will give us sufficient flux in the $3-8 \mu \mathrm{m}$ bandpass.

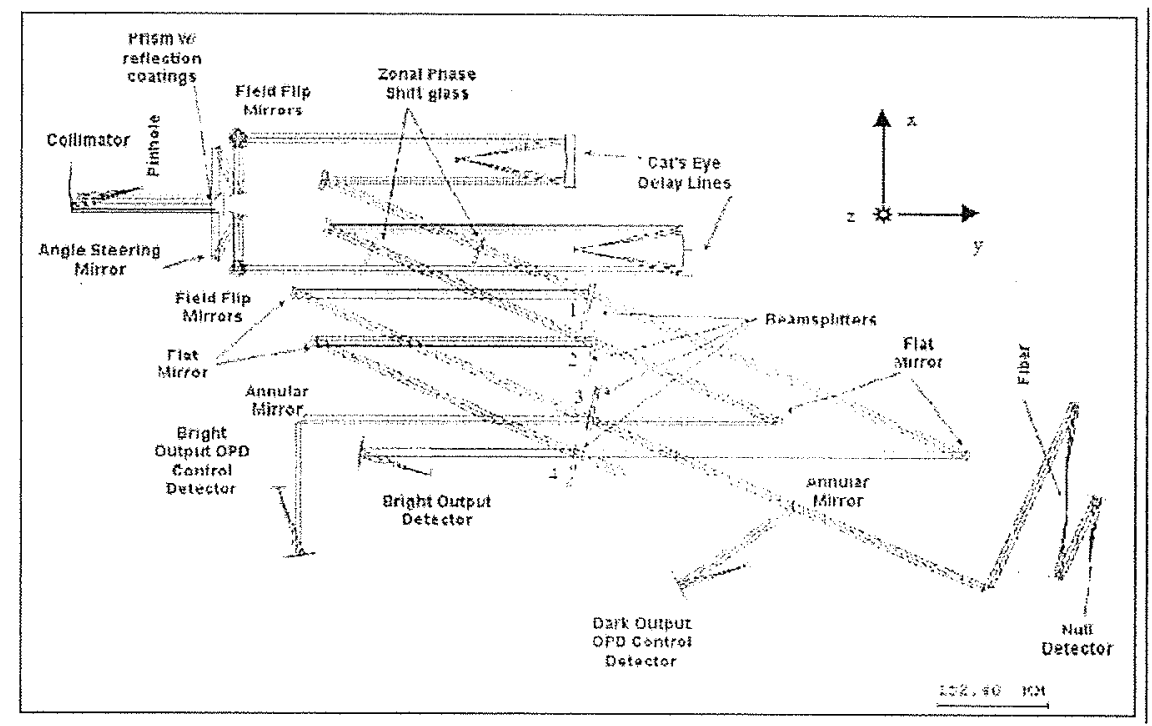

Figure 1: FKSI testbed optical layout

The most significant addition to the testbed in recent months has been the development of the closed loop OPD control system. This system, its components, and its feedback mechanisms are described in detail in the following sections. We have also purchased a rigid, plexiglas enclosure to surround the entire optical table to attempt to mitigate environmental effects such as air turbulence. The implementation of this closed loop control system in concert with the plexiglas enclosure should provide the necessary envirommental stability required to achieve our goal of $10^{4}$ null depth.

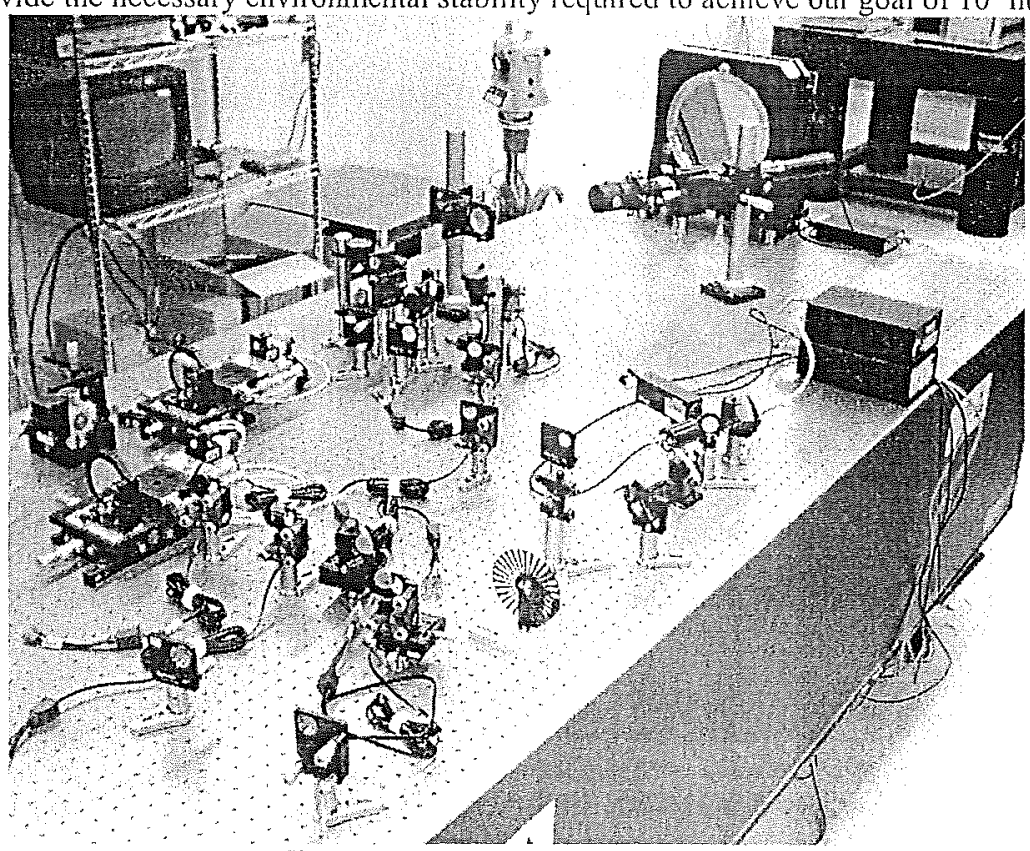

Figure 2: FKSI testbed laboratory setup 


\section{METROLOGY REQUIREMENTS}

Null depth, a critical final output parameter of the testbed integrated test and analysis, is affected by alignment, control, and dynamic stability concerns. An in-depth control system and dynamic jitter analysis has been conducted for the FKSI mission. (See Hyde, et. al., 2004) This analysis consisted of several distinct phases. First, a structural finite element analysis was conducted using a NASTRAN model of the spacecraft bus and instrument payload systems. The second phase of the analysis consisted of development of an optical model and included tracing of ray bundles from the entrance pupils above the siderostats through the optical train to the angle tracker, fringe tracker, and nuller detectors. The third modeling effort included the normal spacecraft attitude control as well as control of many optical elements in the payload. This model, carried out in MATLAB Simulink, includes the spacecraft attitude control system (ACS) as well as the two types of continuous control loops in the payload, the optical path delay (OPD) and the angle steering mirrors (ASM). Finally, the results of the discipline models outlined above were integrated into a single system model. The model integration and subsequent analysis was performed in the Disturbance-Optics-Controls-Structures (DOCS) environment, a suite of tools developed at the Massachusetts Institute of Technology for performing integrated modeling, critical parameter identification, and system design. The influence of each design parameter and anticipated disturbance was explicitly evaluated. Critical parameters were then traded to generate a design that meets requirements optimally with respect to mission cost and risk.

\begin{tabular}{|l|r|}
\hline Source & OPD. nm \\
\hline FT noise & 5.0 \\
\hline ODL noise & 5.0 \\
\hline ACS induced & 7.0 \\
\hline Boom induced & 1.1 \\
\hline RWA induced & 6.0 \\
\hline Margin & 9.4 \\
\hline RSS Total & 15.0 \\
\hline
\end{tabular}

Table 1: Sources of noise and their contributions to the total null loss budget

From these analyses testbed requirements were then derived. Specifically, science drivers require a 0.01 percent null depth that may be allocated across the derived null depth loss contributors; null loss formulae subsequently yield a tolerance on parameter for each subsystem. The high-fidelity analysis allows the budgeted OPD tolerance term to be further broken down into separate component contributions. Finally, these parameters are directly applied to FKSI

\begin{tabular}{|c|c|c|c|c|}
\hline Term & Null loss formula & Tolerance on & Tolerance & Null loss \\
\hline OPD, rms & $(2 \pi z /)^{2} / 4$ & $z$ & $15 \mathrm{Hnt}$ & 0.000073 \\
\hline Tilt, rms & $(\pi D \omega h)^{2} / 8$ & Do & $34 \mathrm{~nm}$ & 0.000012 \\
\hline WFE, rms & $(2 \pi \sigma / \lambda)^{2 / 4}$ & $\sigma$ & $611 m$ & 0.000012 \\
\hline Amplitude match & $1-2 /\left(R^{1 / 2}+R^{-1 / 2}\right)$ & $1-R=1-A_{1} / A_{2}$ & $1.2 \%$ & 0.000004 \\
\hline Pupil area overlap & $1-f_{\text {oretlap }}$ & $1-f_{\text {ouring }}$ & $\begin{array}{ll}N A \% \\
\end{array}$ & NA \\
\hline Polarization match & $\theta^{2} / 4$ & $\theta$ & $0.36 \mathrm{deg}$ & 0.000010 \\
\hline Polarization shift matel & $(\theta / 2)^{2} / 4$ & $\theta$ & $0.72 \mathrm{deg}$ & 0.000010 \\
\hline & & & & 0.000099 \\
\hline
\end{tabular}

Table 2: Sources of noise contributing to the total OPD error budget. 
testbed OPD components as design requirements. The ACS, RWA, and boom noise contributions are root-sum-squared and are equated in the testbed environment to residual seismic and air turbulence perturbations. The resulting requirements on the testbed system are that sensor noise in the fringe detector accounts for about $5 \mathrm{~nm}$ of OPD error. Actuation noise in the delay line fine stage also accounts for about $5 \mathrm{~nm}$ of OPD error.

\section{CONTROL SYSTEM DEVELOPMENT}

Coarse and fine control of the optical path difference (OPD) in the testbed is accomplished by way of two cat's eye delay lines (CEDL), one in each arm of the interferometer. Coarse OPD control is maintained through motion of the entire cat's eye assembly; fine OPD control is achieved by moving the secondary along the optical axis but within the depth of focus of the primary. This two-tier control scheme has been chosen so that the acquisition of the target fringe can be accomplished over wide range of travel by the coarse control mechanism without having to maintain the nanometer accuracy that will be required for the eventual position stability. Once the target fringe has been found by scanning the coarse OPD controller (i.e. OPD $\approx \lambda$ ), the coarse mechanism's motion stops and the fine, closed-loop control takes over to achieve the required nanometer position stability of the CEDL. The main challenge of the CEDL control lies in the fine control where desired control signal strength is close to the noise level of the controller and actuator driver.

The coarse mechanism drives a linear translation stage upon which the primary, secondary, and fine control mechanism are mounted. The coarse mechanism utilizes a direct-drive lead screw driven by a piezoelectric micro-stepping motor, has the travel range of about $12 \mathrm{~mm}$, steps in $160 \mathrm{~mm}$ full step increments with 16 micro-steps/step capability, and is used under open-loop control to find the target fringe (i.e. the central fringe of the white light fringe packet). The science wavelength range for the testbed is $3-8 \mu \mathrm{m}$, so the full step size of the actuator is sufficiently fine to position the mechanism to well within a single wavelength of the targeted central fringe. Once this target fringe is acquired, the coarse control motion can be frozen by powering off the piezoelectric driver. The control of the mechanism is carried out via an RS-232 interface on the testbed control PC.

Figure 3 shows our "first light," open-loop, laser fringe data using the coarse OPD mechanism. Using a custom software interface, we commanded the coarse OPD mechanism to move at a "constant" rate of $3.2 \mu \mathrm{steps} / \mathrm{sec}(32 \mathrm{~nm} / \mathrm{sec})$, its slowest programmable step rate. While the cat's eye was moving, we simultaneously measured its relative motion using a displacement measuring interferometer (DMI), which has a position resolution capability of $1.2 \mathrm{~nm}$, and recorded the fringe intensity at one of the output ports. No attempt has been made to mitigate envirommental disturbances in this data; the visibility is therefore around $88 \%$. The addition of environmental and closed loop path length control will dramatically improve our fringe visibility.

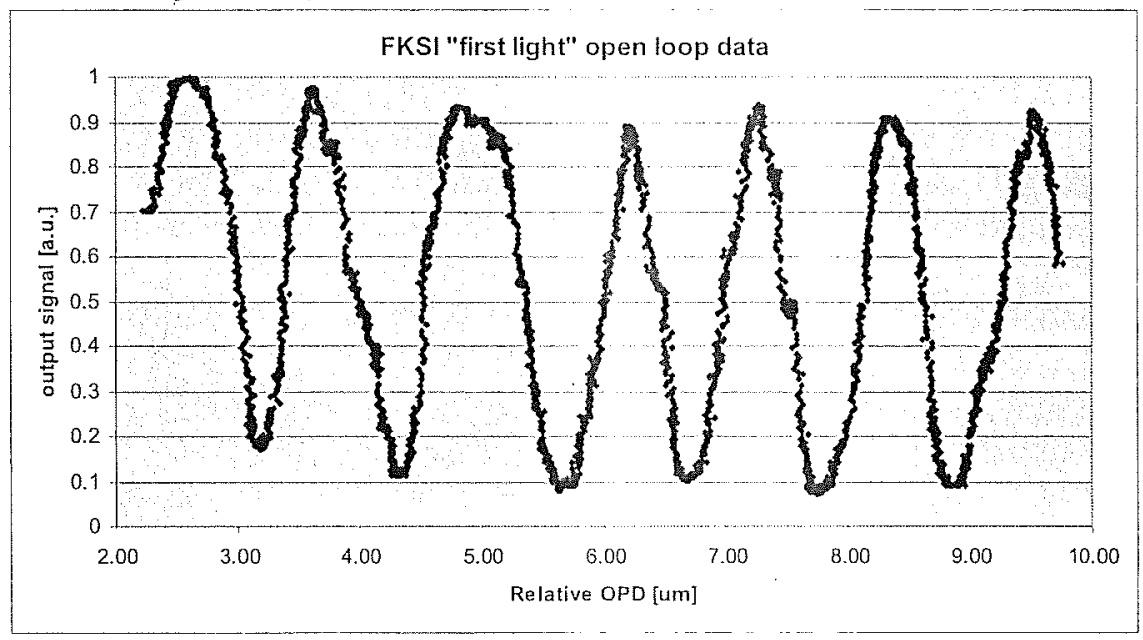

Figure 3: Laser fringe profile using the open-loop coarse OPD control mechanism. The effect of air turbulence on fringe visibility is significant and is the focus of upcoming testbed improvements. 
The fine control mechanism controls a second linear stage carrying the CEDL secondary mirror. A stop and stare dithering technique is used to optimize and track the target fringe while maintaining knowledge of position stability to within $5 \mathrm{~nm}$. The $5 \mathrm{~nm}$ dynamic stability specification is derived from the OPD error budget allocation described above. The fine control actuator that drives the linear stage is a piezoelectric stack and the required drive voltage is $100 \mathrm{~V}$ for 38 um range of motion. In our dithering algorithm we use four step positions, and stare time at each step position depends on the beam signal strength, fidelity of the detector signal, and the detector bandwidth. Currently, the target dither frequency is around $10 \mathrm{~Hz}$ and is limited by the bandwidth of the single element detector electronics.

The integrated intensity measurements at each of these four step positions are used by the dithering algorithm to compute a new central peak value for the target fringe. A new command profile is generated by combining the dithering step profile and this computed center peak. Both feedforward and feedback control algorithms are implemented to optimize the transient and steady state responses of the step motion (see Figure 4). A displacement measuring interferometer (DMI) system is the primary feedback sensor for position sensing. A high precision capacitive sensor is also available with an absolute position sensing capability and will be mainly used for system characterization. The capacitive sensor provides sub-nanometer resolution, and if its repeatability and non-linearity are measured to be

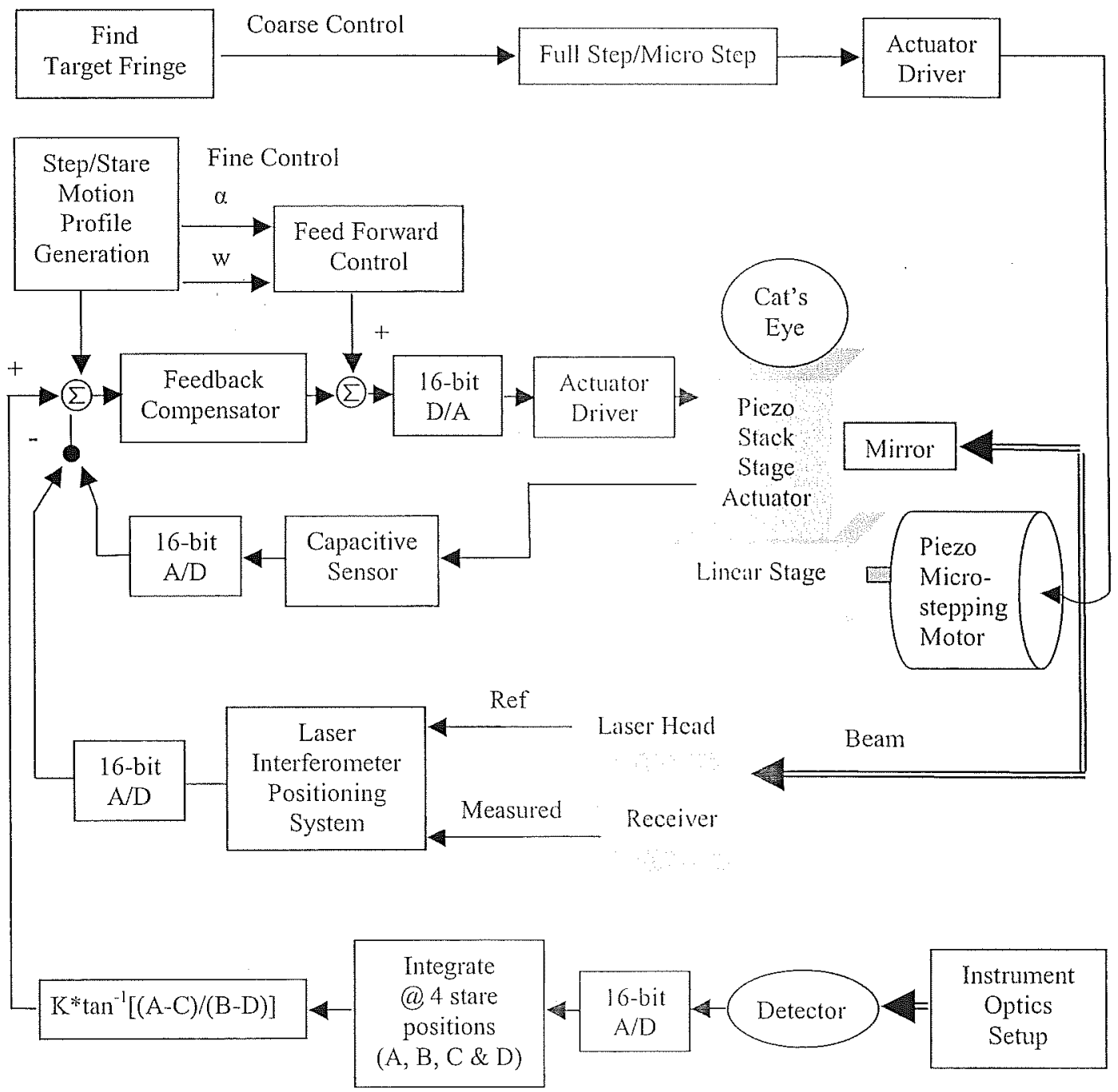

Figure 4: FKSI Cat's Eye Delay Line (CEDL) Mechanisms Control Diagram 
comparable to those of the laser system, it can be used as an alternate position sensor. The control algorithms including the command profile generation, integration of detector signals, dithering center calculation, implementation of feedback/feedforward compensators, sensor calibration and timing sequence are all accomplished in Mathworks' Simulink environment. The C-coded program is generated through Mathworks' Real-Time Workshop and is loaded onto dSpace real-time processor board that operates on TMS320C40 DSPs.

The resolution specification of the DMI system is $1.2 \mathrm{~nm}$ and operates at a bandwidth of up to $12 \mathrm{kHz}$. The range of the DMI actuator drive signal at the output of the D-to- $\mathrm{A}$ interface board is 0 to $10 \mathrm{~V}$, and a driver module delivers the gain amplification of 10 to provide 0 to $100 \mathrm{~V}$ output required by the piezoelectric stack. Our dSpace system utilizes 16 -bit A-to-D and D-to-A converters for its inputs and outputs respectively, so the maximum resolution of the $100 \mathrm{~V}$ drive signal is therefore $0.15 \mathrm{mV}$. Assuming we will utilize the entire $38 \mathrm{um}$ range of the piezo stack, the $1.2 \mathrm{~nm}$ position feedback resolution equates to $0.3 \mathrm{mV}$ for the servo signal. As a result, sub $\mathrm{mV}$ signal noise has the potential to significantly impact the system performance and must therefore be carefully controlled. The estimated throughput rate of the digital control system including the input/output interface is around a few $\mathrm{kHz}$. The bottleneck of the controller is again the detector electronics with its maximum sample rate of $100 \mathrm{~Hz}$. Given the detector bandwidth, the present controller should be able achieve about $20 \mathrm{~Hz}$ of closed loop bandwidth.

Dynamic jitter analysis of the FKSI flight mission requires $5 \mathrm{~Hz}$ closed loop bandwidth to null the OPD error induced by the external disturbances, with the primary contribution coming from reaction wheels. However the calculations in the dynamic jitter analysis ${ }^{4}$ are based on a system utilizing a ditherless quadrature phase detector ${ }^{1}$. The present step and stare dithering control algorithm can accomplish the same end result, but with additional drawbacks including transient response, associated time delay, and possible self-induced jifter contributions in addition to other external (environmental) disturbances. Higher than $5 \mathrm{~Hz}$ closed loop bandwidth is therefore needed for the present testbed to better control the transient behavior and to achieve good disturbance rejection capability.

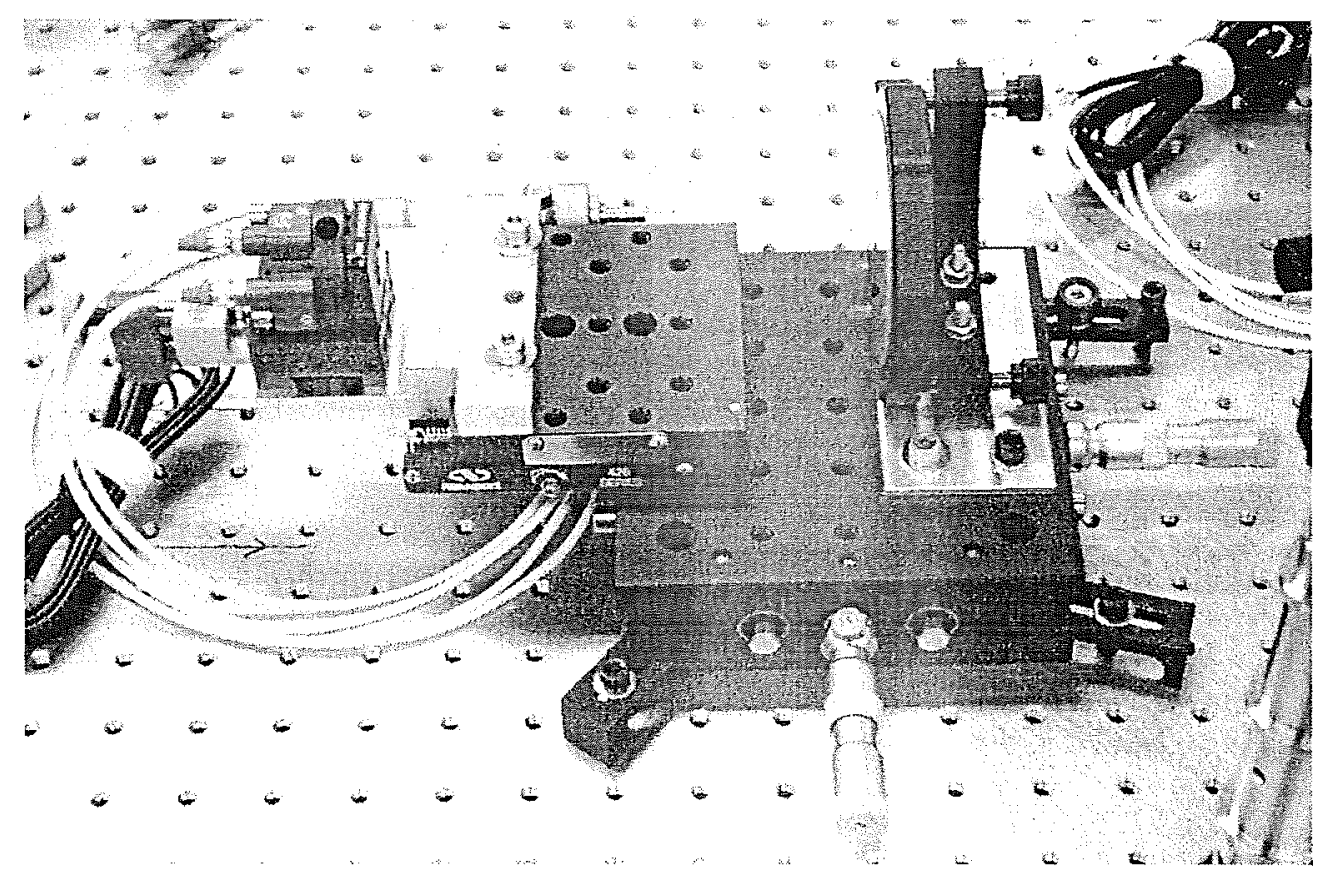

Figure 5: Original cat's eye delay line with coarse and tine delay machanisms. New piezoelectric actuators for more precise OPD control will be installed in the near term to improve testbed performance.

Piezoelectric actuators have been chosen for both the coarse and fine control mechanisms to achieve the high mechanical stiffness, speed, and, in the case of the fine mechanism, to eventually attain high closed loop bandwidth and high disturbances rejection capability. Piezoelectric actuators have been used for space applications for the above mentioned 
reasons and also for their very low power consumption. Most space applications, though, have been in ambient temperature enviromment and cryogenic applications do present additional challenging issues such as temperature dependence, outgassing, insulation material, bonding material and casing. There are ongoing NASA R\&D efforts utilizing piezoelectric actuators in cryogenic environments that could be used in an eventual FKSI flight mission.

\section{FUTURE PLANS}

While we have made significant progress in the past year, we still have a long but well-defined path upon which to travel before we reach our goal of $10^{4}$ mull depth. The primary improvement thit will allow us to reach that goal is automated control of the testbed OPD as described in Section 4. This will incorporate the control system developments as described above, as well as a more precise measure of the actual OPD between the two arms of the interferometer. Currently the DMI allows us to measure the relative position and motion of one of the optical delay lines, but other environmental factors such as changes in the refractive index of air due to temperature or pressure, relative motion of testbed components due to thermal changes, etc, can affect OPD anywhere in the optical path and will not be compensated for by the measurement of a single delay line provided by the DMI. In addition, the dithering technique for measuring the relative optical phase reduces the duty cycle of "science" data acquisition. For these reasons we are planning to implement a ditherless phase detection system that will allow us to remain at or near our null position nearly full time without having to intentionally disturb the system by dithering the delay line.

Such a ditherless phase detection system can be implemented in a variety of ways. Our plan is to construct a "phase plate" of some IR transmissive glass (e.g. ZnSe) such that different regions of the plate will introduce different amounts of optical delay in our science beam. This phase plate would be inserted in one of the two arms of the interferometer, and each of the four quadrants of the plate would introduce a relative phase delay of either $0, \lambda / 4, \lambda / 2$, or $3 \lambda / 4$. By measuring the relative intensities of the four quadrants after combination with the other, unmodified, arm of the interferometer, one can calculate the relative phase difference between the two interferometer arms (see Figure 6).

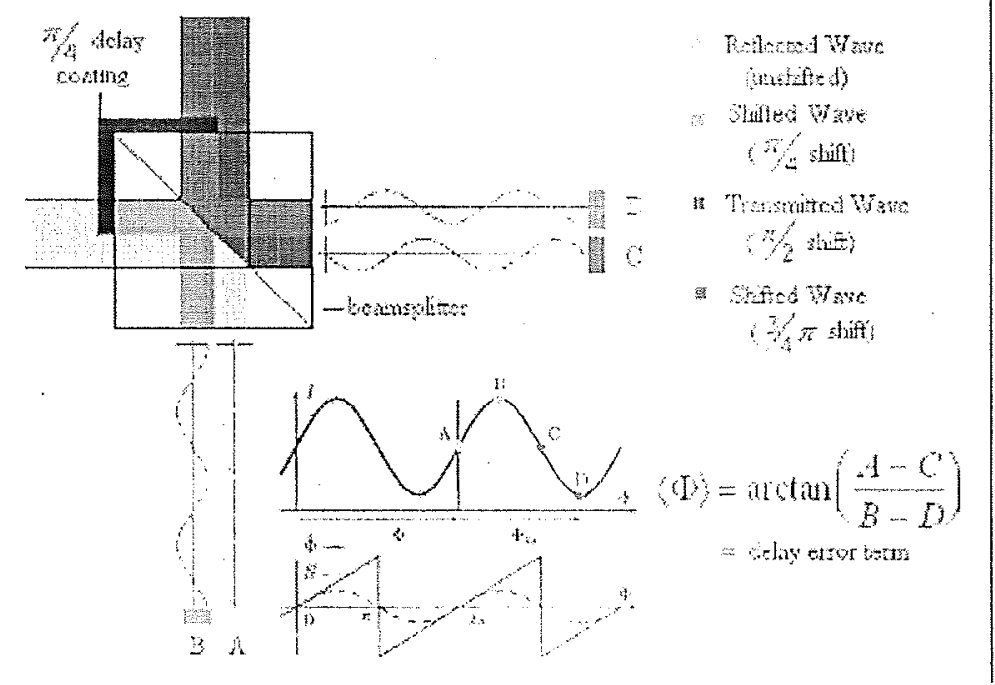

Figure 6: Conceptual schematic for a ditherless quadrature phase detection system. By measuring the intensity of four output beams separated by a relative phase of $\lambda / 4$, we can derive the relative phases of the two incident beams.

Construction of this phase plate can be accomplished in several different ways, all with their own difficulties. The first option is to use an ion etching process to etch away the desired amount of material from a parent until the differential phase delay is as desired. Using this technique there are practical limitations on the relative etch depth $(<5 \mu \mathrm{m})$, but it looks the most promising for our application. Another alternative is to very precisely polish four individual pieces of glass to the prescribed relative thicknesses and optically bond them together into the correct shape. This technique is also challenging, but works well for relative optical delays of 10's to 100's of $\mu \mathrm{m}$. A final technique we have investigated is differentially applying an optically transmissive coating to a flat glass window. In this case the optical delay would be due to a difference in path length through the coating rather than through the glass. Unfortunately the 
generally lower refractive index of the coatings requires a coating thickness that is impractical in most cases, so we have abandoned this approach.

A final consideration with which we will have to contend in the near future is that of atmospheric turbulence. Because we are operating the testbed on a tabletop in the laboratory (i.e. not in vacuum), slight changes in air temperature, pressure, or the introduction of turbulence will cause significant changes in our OPD. For example, our requirement to hold the OPD between the two arms of the interferometer to $<5 \mathrm{~nm}$ requires us to control the temperature of the air path (i.e. the refractive index of air) to better than $0.5 \mathrm{~K}$ (assuming a $1 \mathrm{~cm}$ turbulence cell size). This becomes even more challenging when one considers the amount of electronic equipment required for such a testbed, not to mention human presence in the lab. To mitigate these environmental factors in the near term, we are enclosing the testbed in a COTS plastic enclosure. This enclosure will mitigate much of the short tem and small-scale fluctuations in the non-common air paths of the testbed and limit such changes to timescales for which the control system can compensate.

In the long term we plan to move the testbed to the new GSFC interferometry lab, a newly constructed facility at GSFC slated to open in the Summer of 2006. This new laboratory is a Class 10000 cleanroom and can house one interferometry testbed such as ours in each of its two rooms. The lab will very precisely control temperature, pressure, relative humidity, acoustics, mechanical vibrations, electromagnetic noise, etc. The lab is completely isolated from the rest of the building and has no exterior walls to aid in the environmental control. A separate control room with CCTV will allow us to remotely control all aspects of the testbed in the comfort of a non-cleanroom environment. These improvements will not only help us reach our primary goal of $10^{-1}$ null depth, but also to develop the control system algorithms to be able to hold that null depth for extended periods of time required to complete the science goals of the mission.

\section{CONCLUSION}

The FKSI mission will provide exciting new science opportunities and can serve as a pathfinder mission for such programs as TPF-I/DARWIN, SPECS, and SPIRIT. In anticipation of such a mission, we have constructed a laboratory testbed to develop the techniques and algorithms necessary to accomplish our mission goals. We have recently acquired our "first-light" IR laser fringe data and in the coming months will add closed-loop OPD control and migrate to a broadband IR blackbody source.

\section{ACKNOWLEDGMENTS}

This work was supported in part by NASA Goddard Space Flight Center's Internal Research and Development (IRAD) and Director's Discretionary Fund (DDF) programs.

\section{REFERENCES}

1. R.K. Barry, et. al., "The Fourier-Kelvin stellar interferometer (FKSI): a progress report and preliminary results from our nulling testbed," Proc. SPIE, 5905, 59050Z, (2005)

2. R.K. Barry, et. al., "The Fourier-Kelvin Stellar Interferometer: an achievable, space-bome interferometer for the direct detection and study of extrasolar giant planets," Proc. IAU Colloquium, 200, 119-124, (2005)

3. R.K. Barry, et. al., "The Fourier-Kelvin stellar interferometer," SPIE 6265-55, Orlando, 2006

4. T. Tupper Hyde, et al, "Requirements Formulation and Dynamic Jitter Analysis for the Fourier-Kelvin Stellar Interferometer," Proc. SPIE, 5497, 553-564, (2004) 